\title{
The inherited diseases of haemoglobin are an emerging global health burden
}

\author{
S. Fucharoen, P. Winichagoon \\ Thalassemia Research Center, Institute of Molecular Biosciences, Mahidol University, \\ Nakornpathom, Thailand
}

\begin{abstract}
Thalasseamia is one of the common genetic disorders. A genetic defect causes reduction of the globin chains leading to chronic haemolytic anaemia from birth. The mainstay of treatment is blood transfusion to maintain adequate levels of the haemoglobin. Secondary iron overload in $\beta$-thalassaemia patients is secondary to multiple blood transfusions and increased iron absorption. Excesses iron potentially catalyses free-radicals generation and impairment in cellular function and integrity. Extensive iron-induced injury develops in the heart, liver, pancreas and endocrine system. In regularly transfused patients, in the absence of iron-chelation therapy, death from iron-induced heart failure occurs by the mid-teenage years. Conventional treatment with the parenteral iron chelator desferrioxamine improves mortality but it is too expensive for middle and lowincome countries. Oral iron chelators, such as deferiprone (L1) and deferasirox, appear to be promising, however, they are still too expensive or need special monitoring. Serum ferritin has been used for many years as a guide for chelation therapy. However, recent studies demonstrated that using serum ferritin or liver iron measurements as a monitor of iron-chelation intensive therapy would have been discontinued long before the iron had cleared from the heart. There is evidence of the value of myocardial T2* measurements by MRI for the detection of early cardiac iron overload which cannot be predicted by liver iron or serum ferritin and for the monitoring of iron-chelation therapy. The major problem is the expensiveness of MRI measurement. In conclusion, the problem of iron chelation in low-income countries may be summarized as follows: i) drugs, are not available in every countries that need the medicine, ii) the cost of drugs is too high for most low income countries, iii) there is poor education of doctor, parents/patients and local government about the benefit of iron chelation,
\end{abstract}

Correspondence: S. Fucharoen, Thalassemia Research Center, Institute of Molecular Biosciences, Mahidol University, Nakornpathom, Thailand.

Key words: global health burden hemoglobin disorders.

(C) Copyright S. Fucharoen and P. Winichagoon, 2011

Licensee PAGEPress, Italy

Thalassemia Reports 2011; 1(s2):e1

doi:10.4081/thal.2011.s2.e1

This article is distributed under the terms of the Creative Commons Attribution Noncommercial License (by-nc 3.0) which permits any noncommercial use, distribution, and reproduction in any medium, provided the original author(s) and source are credited.

Parts of this work were presented at the "12th International Conference on Thalassemia and Hemoglobinopathies”, Antalya (Turkey), 11-14 May 2011. iv) there is need for monitoring of its toxicity and adverse drug reaction. In the TIF conference in Dubai, in 2006, a group of experts had agreed to send a document to the D-G of the WHO with a strong request that all chelators (currently available and those that will be available in the future) be designated essential for the treatment of transfusion dependent anaemias.

\section{Introduction/Epidemiology}

Although most monogenic diseases are quite rare, because of selection by malaria genetic blood diseases, particularly the thalassaemias, occur at a high frequency in many tropical populations, and recently it has become apparent that they will pose an increasingly severe health burden for the countries of Asia. Thalassaemia is one of the most common single gene disorders. Approximately 5-7\% of the world's population are carriers, and 300,000 to 500,000 babies with severe forms of haemoglobin disorders are born each year. $\alpha$ - and $\beta$-thalassaemias and some abnormal haemoglobins $(\mathrm{Hb})$ such as $\mathrm{Hb} \mathrm{S}, \mathrm{Hb} \mathrm{E}$ and $\mathrm{Hb} \mathrm{D}$ are common. Globally, about $1 \%$ of couples are at risk of having affected children of which approximately $82 \%$ are sickle cell disorders and $18 \%$ are major thalassaemic diseases. ${ }^{1-3}$

$\alpha$-thalassaemia, $\beta$-thalassaemia and $\mathrm{Hb} \mathrm{E}$, are noted hallmarks of Southeast Asia where the $\alpha$-globin variant, Hb Constant Spring is also prevalent (Table 1). $\alpha$-thalassaemia also has foci in Laos and northern Thailand where it is present at frequencies of $30-40 \%$. Although thalassaemias are most frequent in tropical regions, they have now spread to most countries because of population migrations that that take these abnormal gene with them. The $\alpha$-thalassaemias 2 ( $\alpha^{+}$-thalassaemia) occur across the tropical zone, whereas the $\alpha$-thalassaemias 1 ( $\alpha^{0}$-thalassaemia) are restricted to some parts of Southeast Asia and the Mediterranean. Furthermore, the most serious form of thalassaemia disease, Hb Bart's hydrops foetalis, is almost completely confined to Southeast Asians. $\beta$-thalassaemia is frequent in the Mediterranean countries and parts of Africa, throughout the Middle East, the Indian subcontinent, Southeast Asia, and Melanesia and into the Pacific islands.

Haemoglobin $\mathrm{E}$ is most concentrated at the junction of Cambodia, Laos and Thailand, the so-called $\mathrm{Hb} \mathrm{E}$ triangle where the prevalence of $\mathrm{Hb} \mathrm{E}$ reaches $50-60 \%$. Hb E is also common in certain ethnic groups in Indonesia, Malaysia, Singapore and Vietnam. It has been estimated that homozygous $\beta$-thalassaemia and $\beta$-thalassaemia/Hb $\mathrm{E}$ occurs in Asia at a frequency of 0.24 and 0.25 per 1000 births, respectively.

The haemoglobin $\mathbf{S}$ gene is distributed throughout Africa, the Indian subcontinent and the Middle East where carrier frequencies range from 5 to $40 \%$. Sickle cell patients (homozygous for the $\mathrm{Hb} \mathrm{S}$ gene) may have transient acute painful episodes that occur with varying frequency and severity. They are susceptible to infection with a high mortality rate, especially in children living in low-income countries.

Complex gene-gene interaction leads to over 60 different thalas- 
saemic syndromes including homozygous $\beta$-thalassaemia, $\beta$-thalassaemia/Hb E, Hb H disease and Hb Bart's hydrops foetalis. Many thousands of people with these complex thalassaemia genotypes are living in the Middle East and Southeast Asia. From the above abnormal gene frequencies, it has been estimated that there are many thousands of major thalassaemic cases born in Southeast Asia each year. In Thailand alone, with a total population of about 65 million and an annual birth rate of 850,000 births, there are more than 10,000 major thalassaemic cases born each year. The growing demands on resources makes thalassaemia one of the most important public health issues in the region. However, for the last 30-40 years there has been very little support from most of the local government to improve the quality of life of thalassemia patients and to prevent the birth of new cases. In the $1980 \mathrm{~s}$ the WHO established a panels of experts on thalassaemia, the WHO Working Group, who developed many guidelines for the treatment and prevention of thalassaemia. ${ }^{1}$ However, most of these guidelines were not implemented, and were ignored by most local governments. As a consequence, regional prevention and treatment programs have been largely undertaken by individual experts and researchers who often share experience by the organization of conferences and workshops. In reality thalassaemia is not an emerging public health problem and thalassaemia should be recognized as an Orphan Disease.

\section{Management}

The requirements for patient care are different for thalassaemia and sickle cell disorders. For sickle cell disorders simple primary care including information and support for families, prophylactic antibiotics, and access to hospital treatment for patients with acute complications. Since young children are at the highest risk for complications, successful management of sickle cell disorders requires early identification of affected infants.

In contrast the clinical severity of thalassaemia is very heterogeneous varying from an asymptomatic presentation to a transfusion dependent presentation. For patients suffering from severe cases of thalassaemia or thalassaemia major, the ideal treatment regimen consists of blood transfusion about once a month and effective iron chelation treatment. Regular blood transfusion helps to maintain adequate levels of hemoglobin that results in a good quality of life. Iron overload in $\beta$-thalassemia patients is secondary to either multiple blood transfusions or increased iron absorption or a combination of both. However, there are obstacles associated with this approach, preventing many thalassaemia patients from receiving the treatment and care they need. Firstly, due to inadequate blood supplies, it is not possible to give blood transfusions to all thalassaemia patients. Furthermore, there are financial costs involved in ensuring the safety of the blood for transfusion and the cost associated with screening the blood supply is often more than what the average hospital is able to incur. Secondly, iron chelation treatment for each child costs approximately US\$270 per month. This does not include the cost of approximately US $\$ 800$ for the pump necessary for subcutaneous infusion, which may last only three to five years depending on the care of the equipment. Even though oral iron chelation was recently become available, it is still too expensive or needs special monitoring. Due to the large number of patients and limited medical resources, especially in low-income countries, it is not possible to give optimal blood transfusions to the majority of patients. They receive no or minimal blood transfusions and no iron chelation. Therefore, many thalassaemia patients develop a certain degree of iron overload when they come to 30 or 40 years of life even with minimal blood transfusions. In the absence of iron chelation death from ironinduced heart failure occurs by the mid-teenage years.

The large number of untreated thalassaemic patients develops a
Table 1. Prevalence of thalassaemia in Asia and Australia.

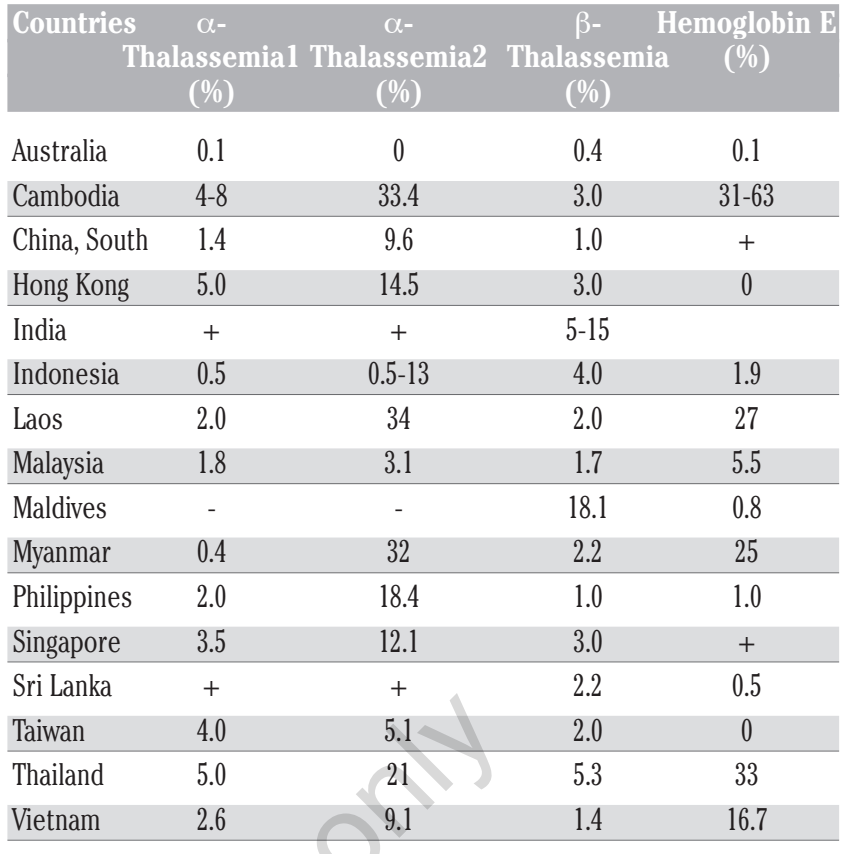

multitude of complications such as infections, hypoxemia, endocrine complication, immunologic abnormalities and trace metals disturbances. Patients who are older than 5 years may benefit from a splenectomy but severe infection has been the cause of death in many splenectomized patients. Although bone marrow or stem cell transplantation (the only cure for thalassaemia) has a more than $75 \%$ success rate, it is rarely available. Even some studies have shown that it is more cost effective to provide stem cell transplantation for thalassaemia before the age of ten the procedure is complex and costly and there are problems in finding appropriate HLA matched donors.

In some countries, many children born with major forms of thalassaemia die either undiagnosed or untreated. Due to a lack of resources, local health centers do not have the facilities to diagnose children born with thalassaemia. If such centers do have the facilities and the capabilities to make proper diagnosis in most cases they do not have the resources to follow-up those children living with thalassaemia. The situation is still that children suffering from major thalassaemia disease die before the age of ten due to anaemia and infection. ${ }^{4}$

\section{Prevention and Control}

Haemoglobinopathies pose a major public health problem throughout Bangladesh, India and Southeast Asia. In Sri Lanka nearly $50 \%$ of transfusion-dependant thalassaemic children were found to have $\beta$ thalassaemia/Hb E. ${ }^{5,6}$ In Thailand approximately 10,000 new cases are born each year while there may be ten times as many in Indonesia. Thus prevention and control of the disease is much more important. Two key issues towards the prevention and control of thalassaemia are ${ }^{1}$ providing the best treatment to the affected cases and ${ }^{2}$ to prevent the birth of new cases. It is timely therefore to set a strategy for the prevention and control of thalassaemia, which includes population screening for heterozygotes, genetic counseling and fetal diagnosis with selective abortion of affected pregnancies. National thalassaemia networks should be initiated to strengthen the prevention and control program of thalassaemia in each country. Prevention strategies are identical for all haemoglobin disorders and include community information, carrier 
detection and genetic counseling for carrier couples followed by prenatal diagnosis. The availability of prenatal diagnosis depends on local economic, medical, societal, religious and legal factors. Carrier screening should lead to the introduction of prenatal diagnosis in countries where it was formerly not available. The objective of carrier detection is to allow an informed choice, and considerable information is available on the actual options to those at risk for thalassaemia. Only a minority of carrier avoids risk by choosing a non- carrier partner. At risk couples with access to family planning tend to restrict family size once they have two healthy children. However, only the availability of prenatal diagnosis can reduce the affected birth prevalence. For the last 20 years it has been shown that prevention and control of thalassaemia is possible in many Mediterranean countries. Success in these programs involves two major components, management and technology. It is expected that during this post genomic era the technologies used in the prevention and control of thalassaemia will be gradually changed to cover the population at large. Carrier detection and characterization of the mutations should be easier and more accurate with less false positives and false negatives and finally it should be cheaper.

With its high prevalence thalassaemia is causing an increasingly severe public health burden for many countries in Asia. ${ }^{711}$ Since thalassaemia carriers have no symptoms and because of the complexity and heterogeneity of the disease it is difficult to diagnose thalassaemia, especially in carriers, at small health care units. Moreover, treatment of severe thalassaemia with regular blood transfusion and iron chelation is expensive. Thus, it is very difficult to persuade international health agencies and governments that thalassaemia is a genuine public health concern compared with the large number of communicable diseases that these countries are handling. However, the inherited haemoglobin disorders have proved to be so important so as to require the development of adequate genetic services for their control and management in the future.

The WHO report adopted by the 57th World Health Assembly in 2004 (Genomics and World Health) recommend that both North/South and local networks should be established as an aid to helping member countries to develop these services. ${ }^{12-14}$ Furthermore, expertise in the diagnosis, control and management of thalassaemia varies considerably from country to country. The concept behind the network would be to establish a working group able to best determine how every country in the network could benefit the most from the shared expertise of this kind. During the last few years a cohesive professional group including the haematologists and geneticists of many countries in Asia have joined hands together as the Asian Network for Thalassemia Control to disseminate good practice in the control and management of thalassaemia in Asia and to provide a central forum for interacting with individual governments and international health agencies to provide support for these objectives. The main objectives are as follows: ${ }^{1}$ the development and dissemination of adequate screening techniques for determining the frequency of the different forms of thalassaemia in Asian countries, ${ }^{2}$ further the evolution of education and screening programs and promote discussion about the possibility of prenatal diagnosis as an interim method for the control of thalassaemia, ${ }^{3}$ the development of more approaches to treatment. In practice, this would entail regular updating meetings about advances in current practice, the establishment of workshops for training in topics like genetic screening, counselling, laboratory diagnosis, control and management and related issues. It could also foster individual partnerships, both for clinical practice and research, within the network. In particular, this would be the most cost-effective way of helping those countries in which these services are not yet available to improve the diagnosis and management of their thalassaemia patients. There seems little doubt that, if the current expertise that is available in some Asian countries were made available to all the countries of the network, these goals would be achieved. It is expected that within the next few years, with a good sup- port, we can help many countries in Asia develop the national program in the prevention and control of thalassaemia. ${ }^{15}$

In conclusion, thalassaemia is causing an increasingly severe public health burden for many countries in Africa and Asia, and there is a need for proper programs directed towards the prevention and treatment of this disease.

\section{References}

1. WHO Working Group. Community control of hereditary anaemias. Bull WHO 1983;61:63-80.

2. Modell B, Darlison M. Global epidemiology of haemoglobin disorders and dervived service indicators. Bulletin WHO 2008;86:480-7.

3. Weatherall DJ. The inherited diseases of hemoglobin are an emerging global health burden. Blood 2010;115:4331-6.

4. Modell B, Berdoukas V. The clinical approach to thalassemia. London: Grone and Stratton 1984.

5. de Silva S, Fisher CA, Premawardhena A, et al. Thalassaemia in Sri Lanka: implications for the future health burden of Asian populations. Sri Lanka Thalassaemia Study Group. Lancet. 2000;355:786 791.

6. Olivieri NF, Muraca GM, O'Donnell A, Premawardhena A, Fisher C, Weatherall DJ. Studies in haemoglobin e $\beta$-thalassaemia. Br J Haematol 2008;141:388-397.

7. Fucharoen S and Winichagoon P. Hemoglobinopathies in Southeast Asia. Hemoglobin 1987;11:65-88.

8. Fucharoen S and Winichagoon P. Thalassemia in Southeast Asia: probelems and strategy for prevention and control. Southeast Asian journal of tropical medicine and public health 1992;23:647-55.

9. Fucharoen $S$ and Winichagoon P. Hemoglobinopathies in Southeast Asia: molecular biology and clinical medicine. Hemoglobin 1997;21:299-319.

10. Colah R, Gorakshakar A, Nadkarni A, et al. Regional heterogeneity of $\beta$-thalassemia mutations in the multi ethnic Indian population. Blood Cells, Molecules, and Diseases 2009;42:241-246.

11. Colah R, Gorakshakar A, Phanasgaonkar S, et al. Epidemiology of $\beta$-thalassaemia in Western India: mapping the frequencies and mutations in sub-regions of Maharashtra and Gujarat. Br J Haematol 2010;149:739-47.

12. WHO 2004. World health assembly resolution on genomics and world health. WHA57.13. WHO, Geneva, Switzerland, [www.who.int/gb].

13. WHO 2006a. Executive board resolution on sickle anaemia. EB117.R3. WHO, Geneva, Switzerland, [www.who.int/gb].

14. WHO 2006b. Executive board resolution on thalassaemia and other haemoglobinopathies. EB118.R1. WHO, Geneva, Switzerland, [www.who.int/gb].

15. Fucharoen S, Winichagoon P. Prevention and control of thalassemia in Asia. Asian Biomedicine 2007;1:1-6. 